\title{
The constant orbital period of FF Ursae Majoris
}

\author{
R. F. Griffin
}

The Observatories, Madingley Road, Cambridge CB3 0HA, UK

e-mail: rfg@ast.cam.ac.uk

Received 27 January 2011 / Accepted 20 October 2011

\begin{abstract}
Gálvez et al. (2007, A\&A, 472, 587) proposed that FF UMa, a double-lined G-type spectroscopic binary, has a variable orbital period. Here they are shown to be mistaken.
\end{abstract}

Key words. stars: late-type - binaries: spectroscopic - stars: individual: FF UMa

In 2007 Galvez et al. (2007a,b) published two papers - one of them in this journal and one in the proceedings of a conference on the chromospherically active double-lined late-type binary system FF UMa (HD 82286). A preliminary orbit, with a period of approximately 3.28 days, had previously been published for it by Jeffries et al. (1995). Gálvez et al. found the most significant property of FF UMa, the one which featured in the titles of both their papers, to be a rapid variation in its orbital period. After presenting their evidence for such a variation - very much larger than has ever been suggested in other cases - they occupied a good deal of their paper suggesting explanations, none of which seems very satisfactory. Here, we demonstrate from their own data that there is no appreciable variation in period.

Gálvez et al. made radial-velocity observations in four observing runs in different seasons. They determined an orbital period, $3.274 \pm 0.054$ days, from one observing run (the last one), but instead of refining it by including the other runs in the solution they adopted a fixed period and then naturally found that there were phasing offsets - or in other words timing errors for the other observing runs. They list those offsets explicitly, in their Table 3, for their three earlier runs and for that of Jeffries et al. They express the offsets both in days and as a proportion of the interval between the relevant run and the last one (the one adopted as the time origin). Naturally the offsets run quasilinearly with time, and all yield proportional timing errors close to $5 \times 10^{-4}$, which are actually listed in Gálvez et al.'s Table 3 .

All that that shows is that they adopted an orbital period that was wrong by that proportion - there is no evidence of any variation in the period. Gálvez et al.'s Table 3 gives the observed $T_{0}$ epochs for each observing run, and the timing offset that each represents. It is easy to work backwards from that information to obtain the epochs corresponding to the ephemeris that they must have used, which are found to differ from the fiducial epoch by exact integer multiples of the period of 3.2735 days that they clearly must have adopted in place of the 3.274 days that they mention. (The integers are 1232, 692, 467 and 215; the 467-times multiple is actually not quite exact - there is probably an arithmetical error, leading to a discrepancy of less than a hundredth of a day.) All that needs to be done to put the orbit to rights is to increase the period by the factor that

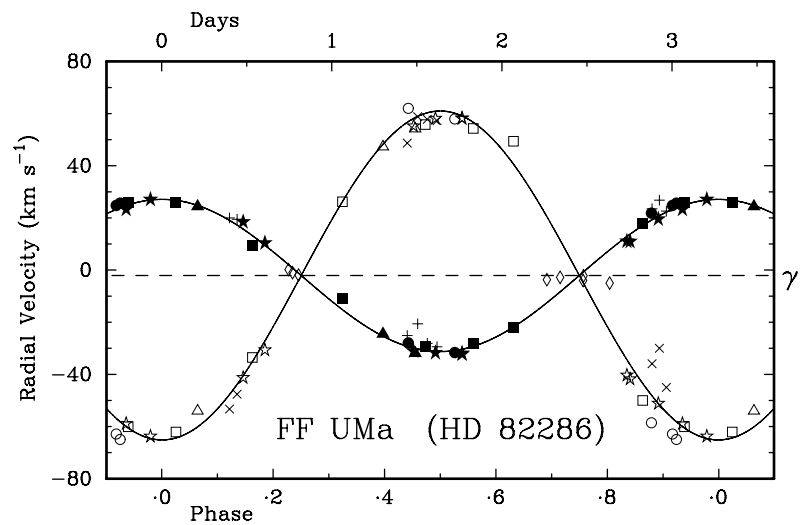

Fig. 1. Observed radial velocities of FF UMa plotted as a function of phase, with the velocity curves corresponding to the adopted orbital elements drawn through them. The radial velocities published by Galvez et al. (2007a) from their four successive observing runs are distinguished by squares, circles, triangles and stars, respectively. Filled symbols are used for the primary star and open ones for the secondary. Observations made near the conjunctions and measured as single-lined are plotted as open diamonds, the individual observing runs not being distinguished; they were not used in the solution of the orbit. The measurements made by Jeffries et al. (1995), which were weighted 1/4 in the solution, are plotted by plusses for the primary and crosses for the secondary. The three secondary velocities near phase 0.9 , which have bad positive residuals, were all made on the same night (the first night), and the significance of their offset should probably not be over-estimated.

Gálvez et al. themselves found, about $5 \times 10^{-4}$. The present writer finds the best value of the period, from the whole ensemble of observations, to be $3.275179 \pm 0.000023$ days - longer than the one that Gálvez et al. must have used by a factor of $5.12 \times 10^{-4}$. There is no occasion to discuss any variation in the period because no such variation is indicated.

For completeness, Table 1 gives the orbital elements now obtained by the writer from the observations of Galvez et al. (2007a) and Jeffries et al. (1995), the latter being given weight $1 / 4$; multiplicatively, the secondary observations have been weighted 1/5. The orbit is illustrated in Fig. 1, in which the different observing runs are distinguished by different symbols. 
A\&A 537, A56 (2012)

Table 1. Orbital elements for FF UMa (HD 82286).

\begin{tabular}{lll}
\hline \hline$P=3.275179 \pm 0.000023$ days & $T_{0}$ & $=$ HJD $2451855.260 \pm 0.009$ \\
$\gamma$ & $=-2.08 \pm 0.35 \mathrm{~km} \mathrm{~s}^{-1}$ & $a_{1} \sin i=1.316 \pm 0.021 \mathrm{Gm}$ \\
$K_{1}=29.2 \pm 0.5 \mathrm{~km} \mathrm{~s}^{-1}$ & $a_{2} \sin i=2.84 \pm 0.05 \mathrm{Gm}$ \\
$K_{2}=63.1 \pm 1.1 \mathrm{~km} \mathrm{~s}^{-1}$ & $f\left(m_{1}\right)=0.0085 \pm 0.0004 M_{\odot}$ \\
$q=2.16 \pm 0.05\left(=m_{1} / m_{2}\right)$ & $f\left(m_{2}\right)=0.085 \pm 0.005 M_{\odot}$ \\
$e \equiv 0$ & $m_{1} \sin ^{3} i=0.183 \pm 0.009 M_{\odot}$ \\
$\omega$ & is undefined in a circular orbit & $m_{2} \sin ^{3} i=0.0846 \pm 0.0033 M_{\odot}$ \\
\multicolumn{2}{r}{ rms residual (unit weight) $=2.05 \mathrm{~km} \mathrm{~s}^{-1}$} \\
\hline
\end{tabular}

\section{References}

Gálvez, M. C., Montes, D., Fernández-Figueroa, M. J., De Castro, E., \& Cornide, M. 2007a, A\&A, 472, 587

Gálvez, M. C., Montes, D., Fernández-Figueroa, M. J., De Castro, E., \& Cornide, M. 2007b, IAU Symp., 240, 214

Jeffries, R. D., Bertram, D., \& Spurgeon, B. R. 1995, MNRAS, 276, 397 